\title{
Maladies génétiques associées aux mutations inactivatrices des récepteurs des gonadotrophines
}

C es dernières années, des progrès considérables ont été faits dans 'identification de nouveaux syndromes correspondant à des altérations génétiques de récepteurs intervenant dans la reproduction. Deux types de mutations germinales ont été décrits pour les récepteurs des gonadotrophines ainsi que pour le récepteur de la TSH qui appartient à la même famille [1-3]: des mutations inactivatrices (pertes de fonctions) et des mutations dites constitutives (gains de fonctions) entraînant une activation du récepteur même en l'absence d'hormone. Les mutations inactivatrices ne s'expriment que lorsque les deux allèles portent chacun une mutation (sujet homozygote ou hétérozygote composite) et donnent naissance à une pathologie à transmission autosomique récessive alors que les mutations activatrices s'expriment de façon dominante à l'état hétérozygote.

Les premières mutations inactivatrices décrites correspondaient à des phénotypes prévisibles, liés à une altération complète de la fonction des récepteurs. Ces mutations naturelles inactivatrices réalisent des invalidations complètes naturelles de la fonction des récepteurs et donnent donc des informations importantes sur le rôle des récepteurs dans le développement et la différenciation des cellules cibles. Cependant, une caractéristique des récepteurs de cette famille est l'existence de pertes de fonction partielles. Nous avons montré que c'est le cas pour le récepteur de la TSH [4] et pour un autre récepteur hormonal couplé aux protéines $G$, le récepteur de la $\mathrm{G} n \mathrm{RH}$ [5]. Ces mutations inactivatrices donnent naissance à toute une variété de phénotypes différents. Bien qu'ils soient peu fréquents, ces tableaux partiels pourraient correspondre à $m / s n^{\circ} 2$, vol. 15, fórier 99 un nombre beaucoup plus restreint d'étiologies assurant un diagnostic génétique plus aisé. En outre, dans le cas des récepteurs des gonadotrophines certaines formes partielles pourraient être accessibles à un traitement de l'infertilité des patients.

\section{Mutations inactivatrices}

du récepteur de la $\mathrm{LH}$

\section{Agénésie et hypoplasie}

des cellules de Leydig

Les premières mutations inactivatrices du récepteur de la LH (LHR] ont été décrites chez des sujets masculins présentant un pseudohermaphrodisme complet (figure 1$)(\mathrm{m} / \mathrm{s}$ $1995, n^{\circ} 5$, p. $774 ; 1996, n^{\circ} 6$, p. 834) $[1,6]$. Il s'agissait du tableau décrit depuis longtemps d'agénésie de cellules de Leydig [7]. Les patients d'apparence féminine consultent pour une aménorrhée primaire avec absence de développement mammaire. L'élévation de la concentration de LH associée à une concentration plasmatique de testostérone effondrée, non stimulable par l'hCG, oriente vers une altération fonctionnelle du récepteur. L'histologie conventionnelle des gonades a révélé dans ces cas l'absence de cellules de Leydig. Ces observations ont permis de montrer pour la première fois que le récepteur de la $\mathrm{LH}$ est impliqué non seulement dans le contrôle de la fonction mais également dans le contrôle de la croissance et de la différenciation des cellules de Leydig. Cette situation rappelle celle du récepteur de la TSH qui est impliqué dans la croissance et la différenciation du tissu thyroïdien.

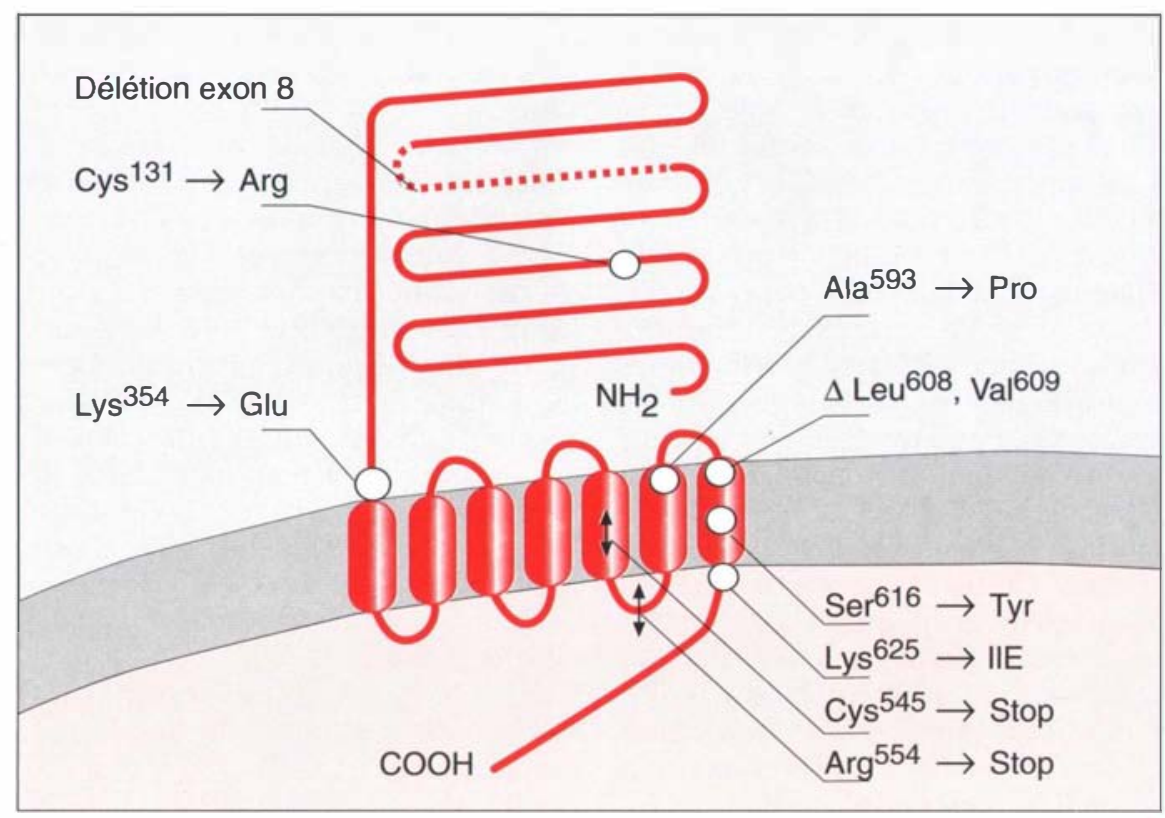

Figure 1. Mutations naturelles inactivatrices du récepteur de la $\mathbf{L H}$. Sont indiquées: des mutations ponctuelles faux sens (o), des mutations non sens $(\leftrightarrow)$, la délétion de deux résidus (4Leu608, Val609) et une délétion extracellulaire de l'exon 8 (trait pointillé). 
Chez l'homme la croissance et la différenciation des cellules de Leydig s'effectuent en trois vagues [8] dont la première semble se situer très tôt puisque la testostérone est détectée chez le foetus humain à la sixième semaine de gestation. La première vague anténatale de croissance de cellules de Leydig semble être indépendante de la LH ou de l'hCG. En effet, chez les patients présentant une perte de fonction du récepteur de la $\mathrm{LH}$, on observe la présence d'épididyme et de canaux déférents. Il y a eu chez ces patients, durant la vie anténatale, une certaine production d'androgènes. Cette observation est confirmée dans un modèle animal : en effet l'invalidation du gène codant pour la sousunité $\alpha$ des hormones glycoprotéiques hypophysaires a été réalisée chez la souris [9]. Les souris invalidées ont également une différenciation de l'épididyme et des canaux déférents. Les autres vagues de croissance et de différenciation des cellules de Leydig ( $2^{\mathrm{e}}$ mois de vie et puberté) sont, en revanche, sous la dépendance de la LH ou de l'hCG et sont donc altérées en cas d'anomalie du récepteur de la LH. Cela entraîne une absence de développement des organes génitaux externes et un phénotype féminin.

Des phénotypes plus modérés associés à des mutations inactivatrices du récepteur de la $\mathrm{LH}$ ont ensuite été rapportés. Il s'agissait de sujets masculins présentant une insuffisance de développement des organes génitaux externes: micropénis associé à un hypospadias ou à une cryptorchidie bilatérale $[10,11]$. Dans ces cas la difficulté a été de corréler les données cliniques aux résultats histologiques et moléculaires. L'histologie des gonades n'a pu être étudiée que chez un des deux enfants et a révélé l'existence de cellules interstitielles fibroblastiques avec absence de cellules de Leydig. Cette observation histologique n'était cependant pas compatible avec l'aspect masculinisé du patient. Cela montre la difficulté d'identifier les cellules de Leydig par les méthodes histologiques conventionnelles. Ces cellules dérivent de cellules mésenchymateuses et sont difficiles à distinguer des fibroblastes. Un autre enfant présentait un phéno- sexuelle à la naissance du fait de mutations inactivant partiellement la fonction du LHR [12]. Nous avons dans ce cas développé une méthode d'étude immunocytochimique des gonades avec des anticorps spécifiques des cellules de Leydig: anticorps monoclonaux dirigés contre le récepteur de la $\mathrm{LH}$, anticorps polyclonaux dirigés contre une enzyme de la stéroïdogenèse $\mathrm{P} 450 \mathrm{c} 17$ spécifique de la voie de biosynthèse des androgènes. Cette technique a permis de montrer clairement la présence, et de quantifier le nombre fortement diminué de cellules de Leydig chez cet enfant (figure 2). Cet aspect était compatible avec la clinique. La masculinisation partielle des organes génitaux à la naissance suggère que pendant le premier trimestre de la grossesse une production de testostérone a eu lieu. Cela est en accord avec le déficit partiel du récepteur muté capable d'induire une certaine stimulation de l'adénylate cyclase pour de très fortes concentrations d'hCG. Pendant les deuxièmes et troisièmes trimestres de la grossesse se produit la croissance des organes génitaux externes. La diminution de la concentration d'hCG plasmatique chez le fotus a été responsable d'une diminution de la stimulation et donc d'une perte de fonction du récepteur muté, expliquant l'ambiguĩté sexuelle à la naissance.

Sur le plan clinique, des altérations fonctionnelles du récepteur de la $\mathrm{LH}$ pourraient théoriquement être associées à tous les intermédiaires allant du pseudohermaphrodisme masculin jusqu'à une infertilité isolée sans anomalie des organes génitaux. Leur étude dans des systèmes d'expression in vitro utilisant des cellules hétérologues pourrait ne pas permettre de mettre en évidence des altérations fonctionnelles mineures des récepteurs.

\section{Chez la femme}

Chez la femme trois mutations inactivatrices du récepteur de la $\mathrm{LH}$ ont été rapportées [1,2]. Dans tous les cas il s'agissait de sœurs de sujets XY présentant un pseudohermaphrodisme masculin, ce qui explique que le diagnostic ait pu être effectué lors de l'enquête familiale systématique.
Le tableau associe une aménorrhée primaire à une concentration plasmatique de LH élevée dissociée de celle des gonadotrophines (FSH normale). L'échographie chez ces patientes montre l'existence d'ovaires kystiques. La biopsie ovarienne effectuée dans un cas a révélé l'existence d'un nombre normal de follicules avec présence de tous les stades de développement folliculaire jusqu'au stade antral. Le développement folliculaire, assuré par la FSH seule, n'est donc pas perturbé. Il existe, en revanche, une absence de développement préovulatoire avec absence de lutéinisation des cellules de la granulosa.

\section{Mutations inactivatrices du récepteur de la FSH}

\section{Chez la femme}

Le syndrome des ovaires résistants aux gonadotrophines a une description ancienne. Entrent dans sa définition l'existence d'une infertilité associée à une élévation des gonadotrophines, la présence de follicules ovariens et une diminution de la réponse des ovaires à une stimulation par des gonadotrophines exogènes. Parmi les étiologies évoquées, une anomalie de fonctionnement du récepteur de la FSH a été suspectée. Une première mutation du récepteur de la FSH a été détectée dans une population finlandaise à fort taux de consanguinité $[13,14]$. Cette mutation extracellulaire entraîne une inactivation complète du récepteur (figure 3). Le phénotype décrit correspondait à une aménorrhée primaire associée à une élévation de la FSH plasmatique avec développement pubertaire variable. Ces jeunes filles présentaient des ovaires atrophiques comprenant des follicules primordiaux ou primaires mais sans développement ultérieur. Cette affection a été décrite comme une des maladies génétiques récessives autosomiques les plus fréquentes en Finlande (incidence 1/8300, fréquence des hétérozygotes $1 / 45$ ). La principale différence avec les femmes présentant une dysgénésie gonadique d'étiologie inconnue était la mise en évidence de follicules à l'examen histologique des ovaires. Ce critère a 


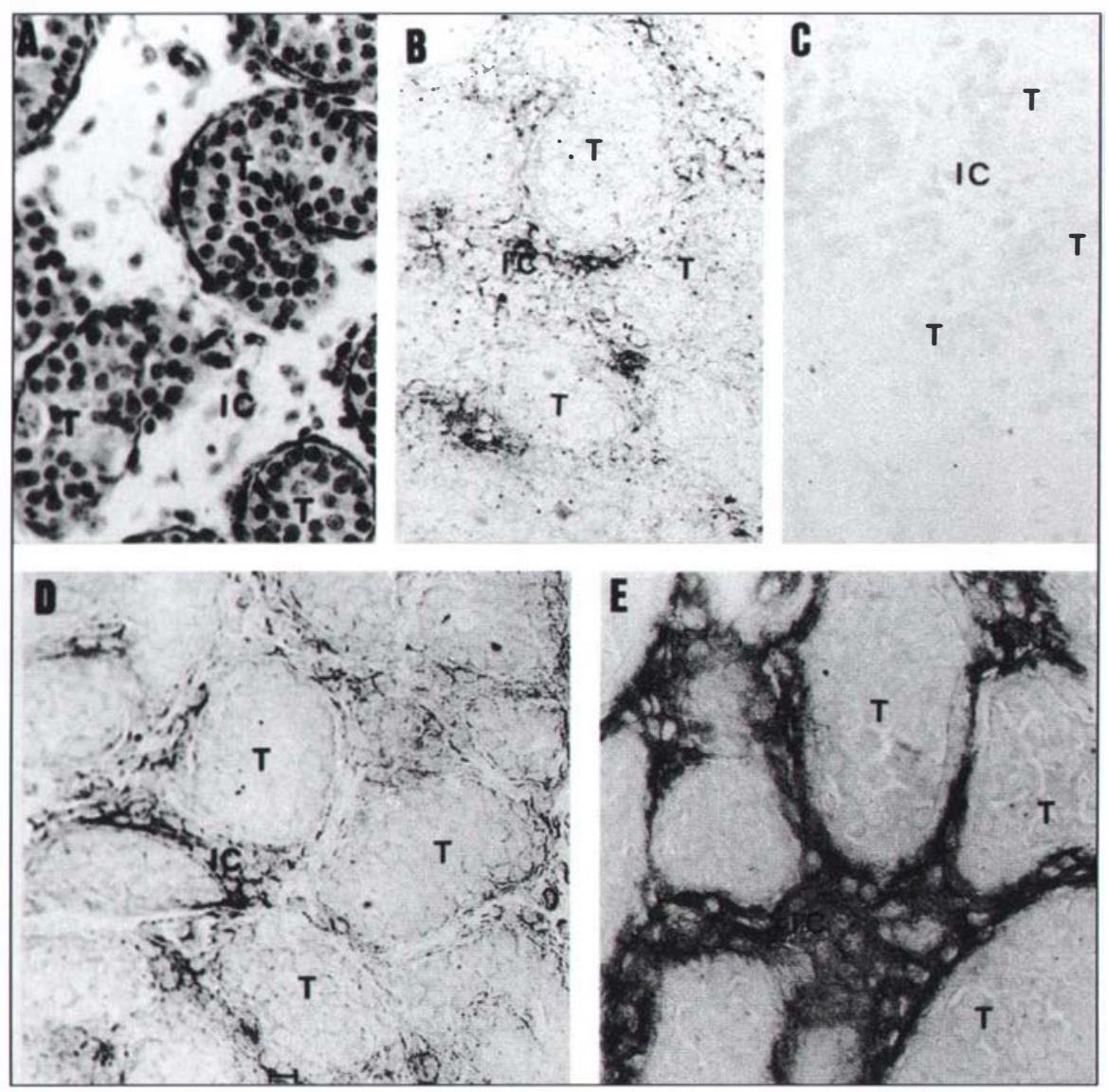

Figure 2. Étude histologique conventionnelle et immunocytochimique du tissu testiculaire d'un patient présentant un pseudohermaphrodisme masculin incomplet dù à des mutations du récepteur de la $\mathbf{L H}$. Un anticorps monoclonal dirigé contre le récepteur de la $\mathrm{LH}$ et des anticorps polyclonaux dirigés contre la P450c17 ont été utilisés [12]. A. Étude histologique conventionnelle: coloration à l'hématoxyline et à l'éosine d'une section de tissu testiculaire montrant plusieurs tubes séminifères immatures $(T)$ et quelques cellules interstitielles $d^{\prime}$ aspect fibroblastique (IC, grossissement $\times$ 400). B. Immunomarquage d'une coupe congelée avec l'anticorps monoclonal dirigé contre le récepteur de la $\mathrm{LH}$. Environ $30 \%$ des cellules interstitielles sont marquées ( $\mathrm{x}$ 400). C. Un anticorps contrôle a été utilisé sur la coupe adjacente du même tissu testiculaire. D. Immunomarquage d'une coupe congelée du même bloc de tissu avec I'anticorps anti-P450c17. Environ 30\% des cellules interstitielles sont marquées (grossissement $\times$ 400). E. Pour comparaison, immunomarquage avec un anticorps anti-P450c17 des testicules $d^{\prime}$ un patient présentant un déficit en 17ß-hydroxystéroïde-deshydrogénase (la même stimulation par I'hCG a été pratiquée avant castration). L'interstitium est rempli de cellules de Leydig. T: tubes séminifères; IC: cellules interstitielles.

donc semblé dans un premier temps pouvoir orienter vers une anomalie du récepteur. Cependant, depuis, plusieurs études de femmes présentant un phénotype similaire ont été conduites dans différents pays et aucune autre mutation du récepteur de la FSH n'a été retrouvée. La situation décrite en Finlande est donc particulière et est très probablement $m / s n^{\circ} 2$, vol. 15 , février 99 patientes présentant un phénotype partiel, par analogie à la situation décrite pour d'autres récepteurs de la même famille $[4,5,10-12]$. Nous avons alors effectivement retrouvé une double anomalie moléculaire du récepteur de la $\mathrm{FSH}$ chez une femme présentant une aménorrhée secondaire après une puberté normale [15]. Une autre différence avec le phénotype décrit dans la population finlandaise était l'existence d'ovaires de taille normale (contrastant avec l'élévation des gonadotrophines). L'examen histologique a montré la présence de nombreux follicules avec un développement folliculaire normal jusqu'au stade antral précoce $(5 \mathrm{~mm})$ (figure 4). Cette observation confirme que le début de la croissance folliculaire nécessite une faible stimulation par la FSH. L'arrêt de développement folliculaire se produit avant le développement préovulatoire, lorsque le follicule nécessite pour croître une forte stimulation par la FSH. L'expression des récepteurs mutés in vitro a montré une altération partielle de la fonction du récepteur, en accord avec le contexte clinique et histologique. L'élévation importante de la LH chez cette patiente est responsable du développement exagéré de la thèque folliculaire* qui peut être délimité par l'étude immunocytochimique des ovaires. Cependant il existe une diminution importante de l'expression du récepteur de la $\mathrm{L} . \mathrm{H}$ au niveau de la thèque lié très probablement à un mécanisme de régulation négative (figure 4). Cela explique l'absence d'hyperandrogénie observée chez cette patiente. Récemment, nous avons retrouvé la présence de deux autres mutations inactivatrices du récepteur de la FSH chez une patiente présentant un phénotype similaire.

Il est important chez ces patientes de pouvoir éliminer d'autres causes à leurs troubles, comme par exemple une ménopause précoce. En effet, on peut espérer dans certains cas pouvoir traiter ces patientes par de très fortes doses de FSH et achever le développement folliculaire.

ment que ce tableau clinique correspond à différentes étiologies qui sont probablement beaucoup plus fréquentes que des anomalies moléculaires du récepteur de la FSH. Nous avons alors choisi d'étudier des
* Structure résultant de la différenciation du stroma ovarien autour des follicules évolutifs. 


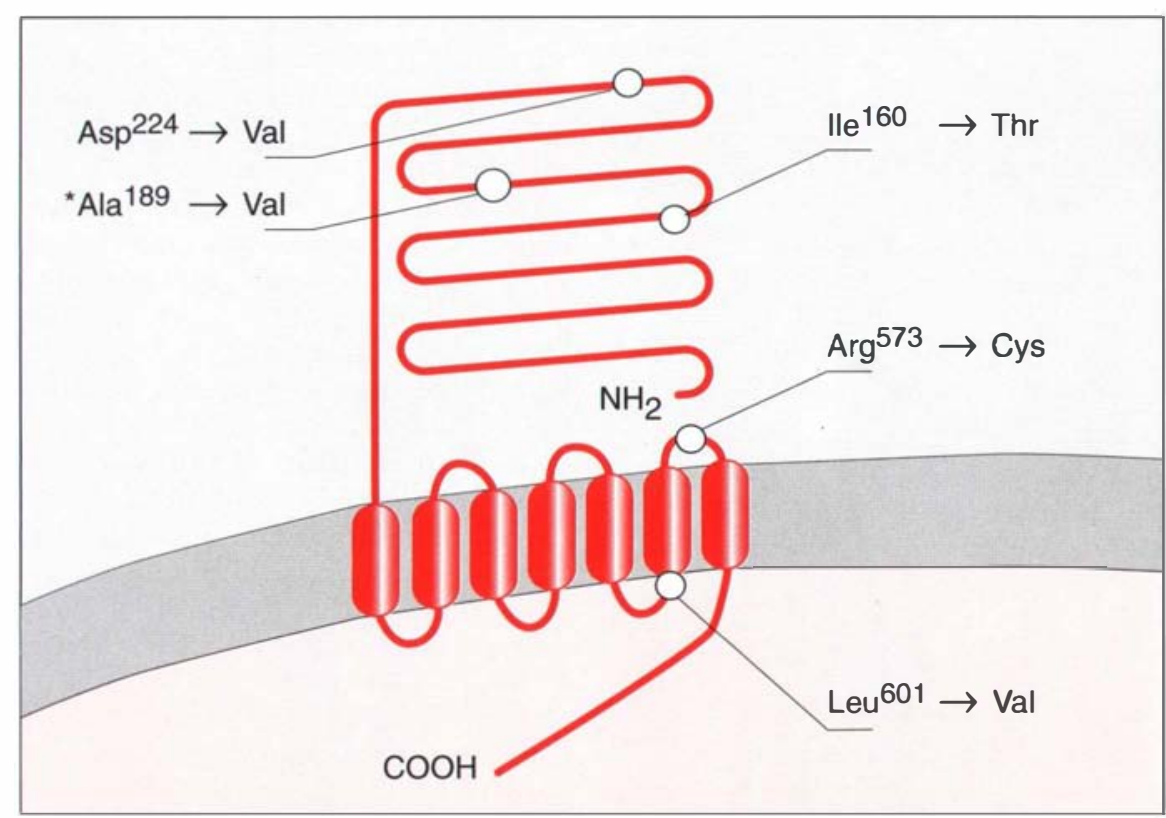

Figure 3. Mutations naturelles inactivatrices du récepteur de la FSH. La mutation homozygote Ala189Val détectée dans la population finlandaise [14] est indiquée par une étoile. Les autres mutations ont été détectées à l'état hétérozygote chez des femmes françaises présentant un phénotype partiel [15].

\section{Chez l'homme}

L'étude de la fratrie des patientes finlandaises a permis de retrouver cinq hommes homozygotes pour l'allèle muté [17]. Tous ces hommes ont eu une puberté normale et présentaient une diminution plus ou moins importante du volume testiculaire, la FSH étant nécessaire à la prolifération des cellules de Sertoli. En outre, ils avaient des troubles variables de la spermatogenèse (pouvant aller jusqu'à l'oligospermie extrême) et de la fertilité, deux d'entre eux étant fertiles $\left(m / s 1997, n^{\circ} 4, p\right.$. 584).

Les conséquences de l'inactivation du gène de la FSH $\beta$ chez la souris sont plus importantes sur la fertilité des femelles que sur celle des mâles[18]. On observe chez les souris femelles un arrêt du développement folliculaire alors que les mâles au gène invalidé sont fertiles avec cependant quelques altérations de la spermatogenèse. En outre, dans un autre modèle animal, la souris $h p g$ déficiente en gonadotrophines, il suffit d'un traitement par androgènes seuls pour pouvoir déclencher et maintenir la spermatogenèse chez les souris mâles [19]. L'ensemble de ces observations a fait suggérer
FSH ou de son récepteur n'empêche pas la mise en route de la spermatogenèse mais est cependant nécessaire au maintien d'une spermatogenèse qualitativement normale. Une mutation de la FSH $\beta$ vient d'être rapportée chez l'homme [20]. Le malade présente une virilisation incomplète, avec un abaissement de la testostérone plasmatique attribuée ici à un défaut d'interaction fonctionnelle paracrine entre les cellules de Sertoli, et les cellules de Leydig. Récemment l'invalidation du gène du récepteur de la FSH a été effectuée chez la souris [21]. Des résultats très similaires sur la fertilité des mâles et des femelles ont été obtenus. Il existe chez les souris mâles homozygotes une diminution significative du nombre et de la mobilité des spermatozoïdes ainsi qu'une augmentation des formes anormales. Une altération de la texture des tubules séminifères est associée qui pourrait provenir d'une anomalie de la lame basale. Chez les souris femelles invalidées, il existe un blocage de la maturation folliculaire au stade de follicule secondaire expliquant leur infertilité. L'analyse d'un nombre plus important de patients présentant des altérations génétiques de la FSH ou de son récepteur est donc nécessaire avant de pouvoir conclure quant au rôle du récepteur dans la fertilité masculine. Les conséquences dans la recherche de nouvelles stratégies contraceptives sont également importantes. S'il est licite de rechercher de nouvelles molécules antagonistes du récepteur pour bloquer le développement folliculaire chez la femme, l'intérêt de ce type de molécules à visée contraceptive chez l'homme reste à démontrer. Le retentissement fonctionnel des mutations naturelles du récepteur de la FSH est apprécié par transfection dans des cellules hétérologues. Il est possible que certaines altérations fonctionnelles ne puissent pas être détectées dans ces systèmes. En effet, au niveau des cellules de Sertoli chez l'homme, par exemple, le récepteur de la FSH a une expression polarisée strictement basolatérale [22]. Une mutation altérant cet adressage polarisé et entraînant une localisation cellulaire apicale du récepteur de la FSH pourrait avoir des conséquences fonctionnelles importantes in vivo alors qu'aucune anomalie fonctionnelle ne pourrait être détectée par expression du récepteur dans les cellules habituellement utilisées qui sont des cellules non polarisées [16]. Cela montre l'intérêt qu'il y a à connaître les mécanismes d'adressage de ce récepteur et, le cas échéant, à utiliser des modèles cellulaires adéquats avant de conclure à l'existence d'un simple polymorphisme.

\section{Conclusions}

Le clonage des gènes des récepteurs des gonadotrophines a ouvert la voie à l'étude de la pathologie génétique correspondante. A l'heure actuelle tous les phénotypes associés à des altérations moléculaires de ces récepteurs ne sont pas encore identifiés. La description de ces nouvelles affections rares est cependant importante à plusieurs niveaux. L'infertilité des malades ayant des altérations incomplètes de la fonction des récepteurs des gonadotrophines pourrait être traitée par de fortes doses d'hormones. Par ailleurs, ces études nous permettent de mieux comprendre in vivo le rôle de ces récepteurs dans les différentes étapes de la croissance et de la différenciation des cellules cibles 


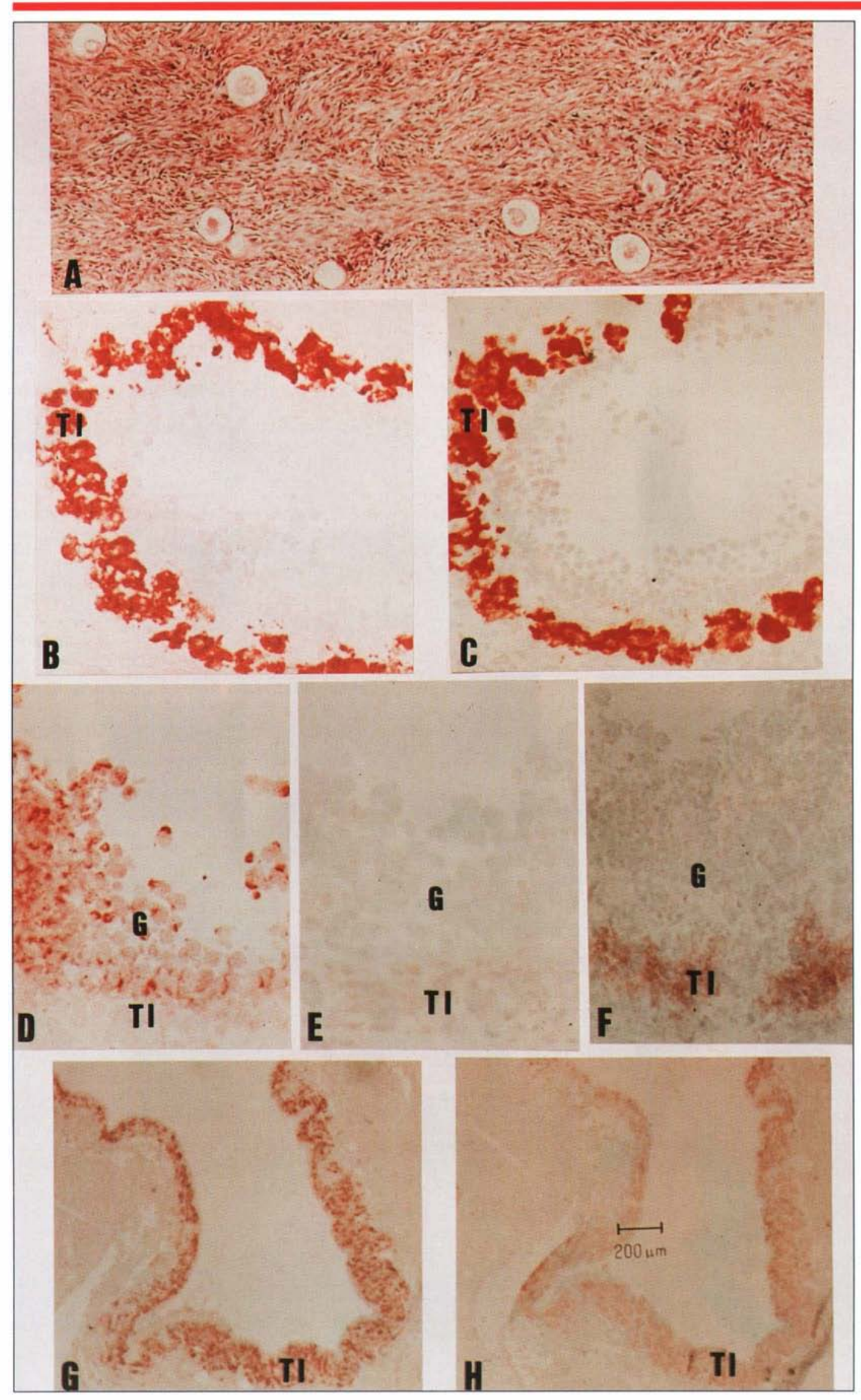

Figure 4. Étude histologique conventionnelle et immunocytochimique des ovaires d'une patiente présentant une aménorrhée secondaire liée à un déficit de la fonction du récepteur de la FSH. A. Follicules primordiaux et primaires: histologie conventionnelle d'une section de paraffine de tissu ovarien (grossissement $\times$ 40). Morphologie et densité normale des follicules. $B, C, D, E, F$. Petit follicule antral $(0,6 \mathrm{~mm})$. Détection immunocytochimique de la $3 \beta-H S D(B)$, de la P450c17 (C) et des récepteurs de la FSH (D) et de la $L H$ (E) sur des coupes congelées. Notez (F) le marquage important des cellules de la granulosa (G) avec l'anticorps antiFSHR, la très faible coloration de certaines cellules de la thèque interne (TI) avec I'anticorps anti LHR et le marquage important d'une thèque interne hyperthrophiée avec les anticorps anti $3 \beta$ HSD et anti P450c17. $\boldsymbol{G}$ et $\boldsymbol{H}$. Grand follicule antral $(\sim 2 \mathrm{~mm})$ : II existe un immunomarquage avec un anticorps anti $3 \beta$ HSD (G) et P450c17 (H). Ce follicule a une thèque interne hypertrophiée (TI) et quelques cellules de la granulosa résiduelles. Grossissement $\times 40$. 


\section{Micheline Misrahi}

Professeur des Universités, praticien hospitalier.

Inserm U. 135, Hormones, gènes et reproduction et laboratoire d'hormonologie et biologie moléculaire, CHU de Bicêtre.

\section{Isabelle Beau}

Assistante hospitalo-universitaire, laboratoire d'hormonologie et biologie moléculaire, CHU de Bicêtre.

\section{Géri Méduri}

Attachée.

Laboratoire d'hormonologie et biologie moléculaire, Hôpital Bicêtre, 78, rue du Général-Leclerc, 94275 Le KremlinBicêtre, France.

\section{Philippe Touraine}

Praticien hospitalo-universitaire.

Service d'endocrinologie et médecine de la reproduction, Hôpital Necker-Enfants Malades, 149-161, rue de Sèvres, 75743 Paris Cedex 15, France.

\section{Claire Bouvattier}

Praticien hospitalier.

Pierre Bougnères

Professeur des universités, praticien hospitalier.

Service d'endocrinologie pédiatrique, Hôpital Saint-Vincent-de-Paul, 82, avenue Denfert-Rochereau, 75674 Paris Cedex 14, France.

\section{Frédérique Kuttenn}

Professeur des universités, praticien hospitalier.

Service d'endocrinologie et médecine de la reproduction, Hôpital Necker-Enfants Malades, 149.161, mue de Sèrres, 75743, Paris Cedex 15, France.

\section{Edwin Milgrom}

Professeur des universités, praticien hospitalier.

Inserm U. 135, hormones, gènes et reproduction et laboratoire d'hormonologie et biologie moléculaire, Hôpital Bicêtre, 78, rue du Général-Leclerc, 94275 Le Kremlin-Bicêtre, France.

\section{RÉFÉRENCES}

1. Themmen APN, Martens JWN, Brunner HG. Gonadotropin receptor mutations. $I$ Endocrinol 1997; 153 : 179-83.

2. Misrahi M, Beau I, Meduri G, Bouvattier C, Ghinea N, Atger M, Vu Hai MT, Loosfelt $H$, Bougnères $P$, Milgrom E. Gonadotropin receptors and the control of gonadal steroidogenesis: physiology and pathology. Bailliere's Clin Endocrinol Metab 1998; 12 : 35-66.

3. Van Sande J, Parma J, Tonacchere $M$, Swillens S, Dumont J, Vassart G. Genetic basis of endocrine disease. Somatic and germline mutations of the TSH receptor gene in thyroid diseases. J Clin Endocrinol Metab 1995; 81 : 4229-35.

4. de Roux N, Misrahi M, Brauner R, et al. Four families with loss of function mutations of the thyrotropin receptor. I Clin Endocrinol Metab 1996; 81 : 4229-35.

5. de Roux N, Young J, Misrahi M, Genet R, Chanson P, Schaison G, Milgrom E. A family with hypogonadotropic hypogonadism and mutations in the gonadotropinreleasing hormone receptor. $N$ Engl J Med 1997; 337: 1597-602.

6. Kremer H, Kraaij R, Toledo SP, et al. Male pseudohermaphroditism due to a homozygous missense mutation of the luteinizing hormone receptor gene. Nat Genet $1995 ; 9: 160-4$.

7. Berthezene F, Forest MG, Grimaud JA, Claustrat B, Mornex R. Leydig-cell agenesis: a cause of male pseudohermaphroditism. $N$ Engl J Med 1976; 295: 969-72.

8. Saez JM. Leydig cells: endocrine, paracrine, and autocrine regulation. Endocrine Rev 1994; 15: 574-626.

9. Kendall SK, Samuelson LC, Saunders TL, Wood RI, Camper SA. Targeted disruption of the pituitary glycoprotein hormone alphasubunit produces hypogonadal and hypogonadal mice. Gemes Dev 1995; 9: 2007-19.

10. Latronico AC, Anasti J, Arnhold IJP, Rapaport R, Mendonca BB, Bloise W, Castro M, Tsigos C, Chrousos GP. Brief report: testicular and ovarian resistance to luteinizing hormone caused by inactivating mutations of the luteinizing hormone-receptor gene. N Engl J Med 1995; 334: 507-12.

11. Laue LL, Wu SM, Kudo M, Bourdony CJ, Cutler GB Jr, Hsueh AJ, Chan WY. Compound heterozygous mutations of the luteinizing hormone receptor gene in Leydig cell hypoplasia. Mol Endocrinol 1996; 10: 987-97.

12. Misrahi M, Meduri G, Pissard S, Bouvattier C, Beau I, Loosfelt H, Jolivet A, Rappaport R, Milgrom E, Bougneres P. Comparison of Immunocytochemical and molecular features with the phenotype in a case of incomplete male pseudohermaphroditism associated with a mutation of the luteinizing hormone receptor. J Clin Endocrinol Metab 1997; 82: 2159-65.

13. Simonı M, Gromolı J, Nieschlag t. I he follicle-stimulating hormone receptor: biochemistry, molecular biology, physiology and pathophysiology. Endocr Rev 1997; 18: 739-73.

14. Aittomäki K, Lucena JLD, Pakarinen P, Sistonen P, Tapanainen J, Gromoll J, de la Chapelle A. Mutation in the follicle-stimulating hormone receptor gene causes hereditary hypergonadotropic ovarian failure. Cell $1995 ; 82$ : 959-68.

15. Beau I, Touraine P, Meduri G, Gougeon A, Desroches A, Matuchansky C, Milgrom E, Kuttenn F, Misrahi M. A novel phenotype related to partial loss of function mutations of the FSH receptor. J Clin Invest 1998; 102 . 1352-59.

16. Beau I, Misrahi M, Gross B, Vannier B, Loosfelt H, Vu Hai MT, Pichon C, Milgrom E. Basolateral localization and transcytosis of gonadotropin and thyrostimulin receptors expressed in MDCK cells. J Biol Chem 1997; $272: 5241-8$.

17. Tapanainen J S, Aittomäki K, Min J, Vaskivuo T, Huhtaniemi IT. Men homozygous for an inactivating mutation of the folliclestimulating hormone (FSH) receptor gene present variable suppression of spermatogenesis and fertility. Nat Genet 1997; 15: 205-6.

18. Kumar TR, Wang Y, Naifang L, Matzuk MM. Follicle stimulating hormone is required for ovarian follicle maturation but not male fertility. Nat Genet 1997; 15 : 201-4.

19. Singh J, O'Neill C, Handelsman DJ. Induction of spermatogenesis by androgens in gonadotropin-deficient ( $\mathrm{hpg}$ ) mice. Endocrinology 1995; 136: 5311-21.

20. Phillip M, Arbelle JE, Segev Y, Parvari R. Male hypogonadism due to a mutation in the gene for the beta-subunit of follicle-stimulating hormone. $N$ Engl J Med 1998; 338: 1729-32.

21. Dierich A, Ram Sairam M, Monaco L, Fimia GM, Gansmuller A, Lemeur M, Sassone-Corsi P. Impairing follicle-stimulating hormone (FSH) signaling in vivo: targeted disruption of the FSH receptor leads to aberrant gametogenesis and hormonal imbalance. Proc Nall Acad Sci USA 1998; 95 : 13612-7.

22. Vannier B, Loosfelt H, Meduri G, Pichon C, Milgrom E. Anti-human FSH receptor monoclonal antibodies: immunochemical and immunocytochemical characterization of the receptor. Biochemistry 1996; 35: 1359-66.

\section{TIRÉS À PART}

M. Misrahı. 